\title{
Special issue on Swarm Cognition
}

\author{
Vito Trianni · Elio Tuci · Kevin M. Passino
}

Received: 13 December 2010 / Accepted: 14 December 2010 / Published online: 23 December 2010

C Springer Science + Business Media, LLC 2010

Swarm Cognition is a novel multidisciplinary approach that encompasses research in neurosciences, cognitive psychology, social ethology and swarm intelligence, with the aim of studying cognition as an emergent collective phenomenon in which perception, attention, decision making and other cognitive processes are brought forth by a multitude of elementary units tightly interacting among each other. Within the Swarm Cognition framework, a broad view of cognition is adopted, so that its definition also includes the behaviour displayed in a distributed system like an ant colony. Indeed, an ant colony can display complex cognitive functions as a result of the interactions among the system components. The parallel with brain activities is straightforward. An ant is part of a colony, much as a neuron is part of a brain. An ant cannot do much in isolation, but a colony is a highly resilient adaptive system. Similarly, a neuron is individually able to only make limited interactions with other neurons, but the brain is capable of highly complex cognitive processes. In other words, both ants and neurons behave/act in perfect harmony with other conspecifics/cells to accomplish tasks that go beyond the capability of a single individual. Out of metaphor, Swarm Cognition aims at studying cognitive processes as the emergent result of the collective dynamics in a distributed system, be the system composed of autonomous agents like ants or basic control units like neurons.

\footnotetext{
V. Trianni $(\bowtie)$

IRIDIA, CoDE, Université Libre de Bruxelles, Avenue F. Roosevelt, n. 50, CP 194/6, 1050 Brussels, Belgium

e-mail: vtrianni@ulb.ac.be

E. Tuci

Department of Computer Science, Aberystwyth University, Aberystwyth SY23 3DB, UK

e-mail: elt7@aber.ac.uk
}

K.M. Passino

Department of Electrical and Computer Engineering, Ohio State University, 2015 Neil Avenue, Columbus, OH 43210-1272, USA

e-mail: passino@ece.osu.edu 
Therefore, Swarm Cognition can be considered part of swarm intelligence, above all for those studies that recognise cognitive processes in the behaviour of distributed systems. In this respect, swarm intelligence can offer a wide range of tools and techniques to understand, study and implement complex behaviour in distributed systems. Swarm Cognition can broaden the perspective of swarm intelligence by applying such techniques to the study of cognitive behaviour, and by exploring the relationship of the behaviour of complex distributed systems with studies in neuro- and cognitive sciences, which are not commonly targeted in the context of swarm intelligence.

This special issue of the Swarm Intelligence journal presents pioneering work in this direction. Following the successful workshop on Swarm Cognition held in July 2009 during the 31st Annual Meeting of the Cognitive Science Society, the special issue solicited high quality contributions that could have a flywheel effect for future research. The special issue opens with the paper "Swarm Cognition: an interdisciplinary approach to the study of selforganising biological collectives" by Trianni et al., which introduces the Swarm Cognition framework by reviewing the most interesting studies in the field and by proposing promising research directions.

The article "Termites as models of swarm cognition" by J. Scott Turner discusses the cognitive function brought forth by the collective behaviour of termites, especially with respect to mound construction and maintenance. Termites are remarkable architects, and this ability influences the cognitive processes of the colony: the complex interactions of the mound with the external environment act as drivers for the behaviour of the termite colony, and are exploited to support its overall behaviour.

Santana and Correia develop a different aspect of Swarm Cognition, by designing a cognitive controller for an autonomous robot. In the article "Swarm cognition on off-road autonomous robots", they describe how a swarm of virtual agents inhabiting the robot visual input can be exploited to recognise obstacles in an extremely parsimonious way. The agents mimic the army ants foraging behaviour, which can focus their group activity in profitable areas. Similarly, virtual agents focus the application of obstacle detection algorithms on portions of the visual field where the obstacle detection is most important for influencing the robot actions. Overall, this distributed system provides the robot with parallel covert attention processes and spatial working memory at a very low computational cost.

Overall, this special issues includes very diverse studies, all linked by the Swarm Cognition perspective. We hope that the reader will find stimulating ideas from reading this special issue. Finally, we would like to thank the editor-in-chief Marco Dorigo for giving us the opportunity to assemble this special issue, and for his continuous support in the complex endeavour of proposing a novel and relatively unexplored research direction.

December 2010

Vito Trianni

Elio Tuci

Kevin M. Passino 